(not unfrequent in private practice also), in which I have found the occasional use of the tincture of digitalis of great benefit. This form of mania has been so exhaustively treated by Dr. Clouston in the Journal of Mental Science for April 1863, that this reference to its treatment will for my present purpose suffice. Time, moreover, warns me to bring wy present remarks to an end.

[Since preparing this communication for the annual meeting of the Association of Medical Offeers of Asylums and Hospitals for the Insane, my attention has been di. rected to a paper read at the thirteenth annual meeting of the British Medical Association in 1862, by Dr. Hand. field Jones, and published in the Britisir Mevical Jourxal, August 23rd, 1862, "Some Remarks upon Re medies and on the Study of their Actions." I fiud some interesting remarks there on the several actions of digitalis in disease, worthy of note by ary one interested in the use of this medicine. I believe there is also a paper by Dr. Christison on the action of digitalis, in some former number of the Edinburgh Monthly Journal. I bave un fortunately not yet had an opportunity of consulting it.]

\section{CASE OF PHTHISIS COMPLICATED WITH PNEUMOTHORAX AND ABSCESS, DESTROYING THE COSTAL CARTILAGES.}

By W. G. Grmson, M.D., Witham, Essex.

Phthisis; Pneumothorax on Right Side; Cavity in Right Lung, communicating with Right Pleura, and also with an Abscess formed to the Left of the Sternum, which burst, destroying the Cartilages and Portions of the Osseous Structure of the Second, Third, and Fourth Ribs. J. B., aged 2:3, in February 1861 laboured under an attack of typhoid fever complicated with pneumonia of both lungs. From this he partially recovered; the pneumonic symptoms were relieved; but he remained in a weak cachectic state, with signs of tubercular deposits in the apices of both lungs.

In August of the same year, after several pleuritic attacks upon the right side, with some pneumonia, the tubercles began to soften; and he expectorated a consi. derable quantity of thick purulent matter. Under good nourishment and medicines, his bealth improved, and he regained to a great extent his ordinary appearance as to flesh, etc.; still he gained little strength, and suffered from frequent attacks of pleurisy on the right side.

In the early part of January 1862, while labouring under an attack of pain in the right side, during the act of coughing, his breathing became very difficult; and, upon examination, signs of pneumothorax of the right pleura presented themselves. In the course of a day or two, a circumscribed red spot (of about the size of a shilling ) appeared on the left side of the sternum, and rather to the upper edge of the fourth costal cartilage: this, however, gradually disappeared, and was succeeded by considerable swelling immediately over the costal cartilage of the third rib, and about an inch and a half to the left of the sternum. In the centre of this swell. ing was a red spot of about the size of a florin, with fluctuation beneath, a considerable amount of pulsation, and empliysema of the surrounding subcutaneous areolar tissue. Under the application of poultices, the skin gradually gave way. 'The tumour burst; and a quantity of dark, grumous, offensive pus, mingled with bubbles of air, was discharged with some relief to the dyspnœa, and followed by the cessation of violent vomiting, which had been of frequent occurrence since the appearance of the spot over the fourth costal cartilage. There were signs of lateral tubercle in the left lung, but no active mischicf in that organ.
The patient continued in a state of hectic, and gradually sank exhausted on June 19 (h, $186 \%$.

Post Mortem Examination. The body was much emaciated. On the left side of the sternum there was a hole in the integument exposing the costal cartilage of the second rib. A probe introduced passed but a very short distance in a direction from the sternum; but, upon passing the probe rather beneath the left side of the sternum, it readily entered a sinus leading towards the right side of that bone.

On making an incision along the sternum, and re. flecting the integument on each side, the muscles on the right side were hardly perceptible, partly owing to their wasted condition, and partly to loss of colour in the muscular fibre. The muscles on the left side, although wasted, were of good colour. Beneath and immediately around the opening in the integument above mentioned, there was an abscess involving the costal cartilages of the second, third, and fourth ribs. The cartilage of the second rib was only slightly implicated; but the portion of rib immediate to it was denuded of pericstenm and carious to the extent of an inch and a half to two inches. The cartilage of the third rib was much destroyed, and a considerable portion of the rib was entirely gone. The entire cartilage of the fourh rib and about two inches of the osseous structure were destroyed and gone. 'The sternum in the vicinity of each of the above named cartilages was more or less implicated.

The abscess, apparently about three inches laterally by four inches longitudinally, contained a quantity of offensive, dark coloured, purulent matter; and, upon examination, was found to be external to the pleura of the left lung, the wall being formed by a dense unyielding tissue, save at a part about the junction of the third costal cartilage with the sternum, where the probe passed readily in a direction upwaids, beneath and towards the right side of the sternum.

On reflecting the sternum carefully, it was found that a thin flap of the right lung was very firmly adherent to its posterior surface. The portion of lung was riddled with softened tubercle, and the probe was found to have passed along a sinus leading to a cavity which communiaated with the right pleura.

The upper portion (about two-thirds) of the right lung was destroyed hy softened tubercle, adherent to the costal pleura, and riddled with cavities; while the lower portion was collapsed and lying close to the spine. The pleura, on its pneumonic aud costal surfaces, was covered with a tiick, vellowish brown, purulent matter. The upper part of the left lung was tiickly studded wiih tubercle in a latent state.

Death from Chrorororm. At the London Hospital on Saturday last, an inquest was held on John Savage, aged 41 years, a mariner, who died from inhalation of chloroform. The deceased had a compound fracture of the right arm, not connected with the joint; and the joint was to be excised. Two drachms of chloroform were administered to Savage, who took it very well at first; then his pulse became feeble. Galvanism and other remedies were applied for half an hour, but with a negative result, and deceased died. Mr. Spence said that the post mortem examination showed the heart was fatty and weak in structure, but no examination during life would have discovered that fact. 'The jury returned a verdict that the deceased died from the administration of chloroform, and that it was properly administered.

European Troors in Burdrah. It has been decided that in future, European troons may be safely sent to liangoon without any previous sojourn in India.

Alleged Uniealthiness of Madras. The mortuary returns show, that for some reason or other, Madras is yearly becoming a more unhenlthy resilence. 